\title{
Morte SÚbita POR ROTURA CARDÍACA DEVIDO A SEVERA INFILTRAÇÃo GORDUROSA DO MIOCÁRDIO. RELATO dE CASO E REVISÃo dA LITERATURA
}

\author{
Rogério L. EISELE
}

Eisele, Rogério L. Morte súbita por rotura cardíaca devido a severa infiltração gordurosa do miocádio. Relato de caso e revisão de literatura. Saúde, Ética \& Justiça, 4(1/2):56-8, 1999.

Resumo: O autor apresenta um caso extremamente raro de morte súbita causado por rotura cardíaca devido a severa infiltração gordurosa do miocárdio. O paciente, um homem de 49 anos, foi encontrado morto embaixo de uma ponte, não havendo sinais externos ou indícios de violência. Na autópsia constatou-se um tamponamento pericárdico causado por uma ruptura cardíaca da parede do átrio direito. O estudo histopatológico da lesão demonstrou uma severa infiltração gordurosa do miocárdio, sem sinais de isquemia cardíaca adjacente ou doença coronariana. A maior parte dos casos de morte súbita estão relacionados com doença coronariana ou arritimias. Embora a infiltração gordurosa já seja bem conhecida há algum tempo, é surpreendente observar que publicações sobre este tema tem sido excepcionais durante os últimos decênios. É possível que certo número de casos de obesidade ou morte súbita cardíaca sejam, na verdade, casos de lipomatose cardíaca que não foram diagnosticados como tal. A infiltração gordurosa do miocárdio, se severa, pode ser causa de disfunções cardíacas graves e, ocasionalmente, morte súbita.

Unitermos: Morte súbita cardíaca. Lipomatose/mortalidade. Ruptura cardíaca/mortalidade.

\section{Introdução}

G rande parte dos casos de morte súbita cardíaca podem ser atribuídos a fenômenos de instabilidade elétrica ou doença cardíaca isquêmica. Recentemente vem atraindo muito interesse devido a casos noticiados entre jovens e desportistas. Na maior parte dos casos foram encontrados lesões coronarianas ou arritimias.

Os autores apresentam um caso de morte súbita devido a rotura cardíaca por severa infiltração gordurosa da parede miocárdica. Dentre as causas de rotura cardíaca, segundo a literatura, estão o infarto do miocárdio complicado, traumatismos torácicos, inclusive massagem cardíaca vigorosa e apenas um caso conhecido de rotura devido a severa infiltração gordurosa 5 .

Os autores descrevem com detalhes os achados patológicos, e chamam a atenção sobre esta patologia, talvez não tão rara, mas subdiagnosticada, sua apresentação e os possíveis mecanismos envolvidos.

\section{Descrição do caso}

E.O.V., 49 anos, masculino, 1,53 m, não obeso, motorista, foi encontrado morto embaixo de uma ponte, não havendo sinais externos ou indícios

\footnotetext{
*Médico Legista do Instituto Médico Legal de Londrina, PR. Patologista do Hospital Universitário da UEL, Londrina, PR. Endereço para correspondência: Rogério L. Eisele - Cx. Postal 8015 - Londrina, PR - CEP: 86010-990
} 
Eisele, R.L. Morte súbita por rotura cardíaca devido a severa infiltração gordurosa do miocárdio.

de violência, ou sinais de manobras de reanimação cárdio-pulmonar.

$\mathrm{Na}$ autópsia foi constatado tamponamento cardíaco com $400 \mathrm{ml}$ de sangue vivo, devido a uma rotura da parede atrial direita, medindo $1 \mathrm{~cm}$ de diâmetro, localizada próximo à transição átrioventricular. O coração era de tamanho e formas normais, pesando 340 gramas, com moderada quantidade de tecido adiposo na superfície. Coronárias pérvias, parede delicada. Demais órgãos sem alterações significativas.

\section{Microscopia}

Os cortes histológicos da área de rotura demonstraram a parede miocárdica severamente infiltrada por tecido adiposo entremeando e substituindo os feixes musculares em toda a espessura da parede e fibras cardíacas residuais com atrofia. Observa-se uma área de solução de continuidade com tecido adiposo nas bordas, com presença de hemorragia em meio à gordura.

O exame histológico das áreas adjacentes, dos ventrículos direito e esquerdo, não demonstraram sinais de isquemia ou de infarto do miocárdio.

Coleta de sangue para exame de dosagem alcoólica (cromatografia gasosa - Head Space) revelou $3.15 \mathrm{~g} / \mathrm{l}$.

\section{Discussão}

A infiltração gordurosa consiste de um acúmulo de tecido adiposo intersticialmente no miocárdio, geralmente associado a uma excessiva quantidade de tecido adiposo no epicárdio; ocorrendo mais comumente na parede anterior do ventrículo direito, afetando ainda, a parede do átrio direito, ou o septo interventricular, onde pode envolver o nó sino-atrial e o nó átrio-ventricular, podendo causar arritmias ${ }^{1}$. Pode ocorrer como lipomatose ou lipoma intra atrial ${ }^{2,3}$, esta última tendo sido descrita em associação com a doença de Uhl' .

A infiltração gordurosa ocorre mais freqüentemente em pacientes com obesidade, porém, em geral, a lesão não causa manifestações clínicas significantes, mesmo quando a infiltração é severa. Alguns autores, afirmam que a lipomatose pode levar a uma insuficiência cardíaca $^{9}$, embora as fibras de setores não comprometidos possam fazer uma hipertrofia compensatória ${ }^{1}$. Em casos extremos pode ocorrer a rotura da parede ${ }^{5,7}$.

A substituição do miocárdio por tecido adiposo se realiza através da transformação do tecido conjuntivo reticular em adiposo com uma atrofia simples e depois numérica das fibras miocárdicas. Não deve ser confundida com o "coração de aspecto tigróide", proveniente da degeneração gordurosa (esteatose cardíaca), em que a gordura está presente no interior das fibras miocárdicas; a qual ocorre em situações de hipóxia prolongada, por anemia profunda, miocardites, difteria, etc.

Embora não se tenha pleno conhecimento dos fatos que antecederam ao momento da morte, nem registros cardiográficos, que nos auxiliem na exata interpretação dos achados; os níveis elevados de alcoolemia $(3,15 \mathrm{~g} / \mathrm{l})$ são fortemente sugestivos de que houve uma elevação dos níveis pressóricos intracardíaco, resultando na rotura da parede miocárdica no local de maior fragilidade: a área de infiltração gordurosa. 
Eisele, R.L. Morte súbita por rotura cardíaca devido a severa infiltração gordurosa do miocárdio.

\section{Conclusão}

Este é um caso raro de alteração cardíaca levando a morte súbita. Alertamos para a atenção diagnóstica cardíaca, patologia talvez não tão rara, mas subdiagnosticada, talvez confundida com obesidade.

A inclusão deste diagnóstico diferencial poderá contribuir no trabalho de desvendar os possíveis mecanismos de disfunção cardíaca envolvidos dentre os numerosos casos inexplicados de morte súbita.

Eisele, Rogério L. Sudden death caused by cardiac rupture due to severe fatty infiltration in the right atrial of the myocardium. Saúde, Ética \& Justiça, 4(1/2):56-8, 1999.

Abstract: The autor presents an extremely rare case of sudden death caused by rupture cardiac due to severe fatty infiltration in the right atrial myocardium. The patient, a 49-year-old man, he was found dead under a brigdge, na he had no evidences of violence or chest trauma. The autopsy showed a pericardial tamponade caused by a cardiac rupture of right atrial myocardium. The histopathologic study of lesion demonstrate a severe fatty infiltration of myocardium, whithout signals of adjacent cardiac ischemia or coronary disease. Although fatty deposits in the heart have been recognised for some time, it is surprising to find that there have been extremely few publications on this subject during the last ten years. Its possible that a certain number of cases of obesity or cardiac sudden death, are really cases of lipomatosis of the heart wich have not been recognised as such. Fatty infiltration of the myocardium, if severe, can be a cause of seious cardiac dysfunction or, ocasionally, sudden death.

Keywords: Death, sudden, cadiac. Heart rupture/mortality. Lipomatosis/mortality.

\section{Referências Bibliográficas}

1. Henriquez, A.; Chuaqui, B. Un caso de lipomatosis del sistema excito-condutor y muerte subita. Rev. Med. Chil., 11:447-50, 1983.

2. Cladellas, M. et al. Endomyocardial diagnosis of cardiac lipomatosis. Cathet. Cardiovasc. Diagn., 13:269-70, 1987.

3. Klintschar, M. et al. Massive injury to the heart after attempted active compression-decompression cardiopulmonary resuscitation. Int. J. Legal Med., 111(2):93-6, 1998.

4. Kusano, I. et al. Cardiac rupture due to severe fatty infiltration in the right ventricular wall. J. Forensic
Sci., 36(4):1246-50, 1991.

5. McGee, J. Oxford textbook of pathology. London, 1994.

6. Cotran, R.S. Robbins - pathology basis of disease. Philadelphia, 1994.

7. Letac, B. et al. Fatty infiltration of the heart and Uhl's disease. Arch. Mal. Coeur. Vaiss., 70(1):p.10713, 1977.

8. Bonhorst, D. Morte súbita cardíaca: definição e mecanismos. Rev. Port. Cardiol., 13(7-8):609-14, 1994.

9. Virmani, R. Cardiovascular pathology. Philadelphia, 1991. 